\title{
A NEW SPECIES OF GUATEMALIA STEYSKAL (DIPTERA, SCIOMYZIDAE) ${ }^{1}$
}

\author{
Luciane Marinoni $^{2}$
}

\begin{abstract}
Guatemalia nigritarsis, sp.n. from Honduras is described and the male terminalia illustrated.

KEY WORDS. Snail-killing flies, Guatemalia, Sciomyzidae, Diptera
\end{abstract}

Steyskal (1960) described Guatemalia, a monotypic genus, with $G$. hubbelli based on males from Guatemala (Panajachel). The same author (1966) examined the types, males and females from Mexico (Guerrero) and Guatemala (Panajachel) of Tetanocera straminata Wulp, 1897 and recognized it as a synonym of his Guatemalia hubbelli. Here is described a second species.

\section{Guatemalia nigritarsis, sp.n.}

Figs 1-7

General coloration: mostly dark brown with yellowish and silvery pruinescence; bristles blackish.

Head. Second antennal segment about half of the face length. Face orange-brown, smooth and shiny; strongly concave in profile. Parafacial silvery-pruinose with a black spot at the limit with the frons. Fronto-orbital plate with an elongated and black spot. Ocellar triangle rounded by a black spot; ocellar bristles well-developed, proclinate and divergent; post-ocellar bristles longer and stronger than the ocellar; inner and outer vertical bristles similar to the post-ocellars; one weak orbital bristle about one third of the post-ocellars.

Thorax. Humeral calli and notopleuron with yellow pruinescence. Humeral and presutural bristles similar to the notopleural. Inferior third of the anepisternum with a silvery-pruinose stripe. Anepisternum and anepimeron bare. Subalar sclerite without vallar bristles. Mesonotum and scutellum with two yellow stripes. Legs mostly yellowish; fore and middle coxae silvery-pruinose; femora and tibiae darkish at distal ends; tarsal segments wholly darkish. Wings brownish, hyaline and white spotted.

Male terminalia (Figs 1-7). Post-abdomen asymmetrical. Epandrium and 8 th sternite symmetrical and well developed. Syntergosternite $6+7$ present and well-developed on left side where it bears the 6th and 7th spiracles. 6th and 7th

1) Contribuição número 770 do Departamento de Zoologia, Universidade Federal do Paraná.

2) Departamento de Zoologia, Universidade Federal do Paraná, Caixa Postal 19020, 81531-970 Curitiba, Paraná, Brasil. 

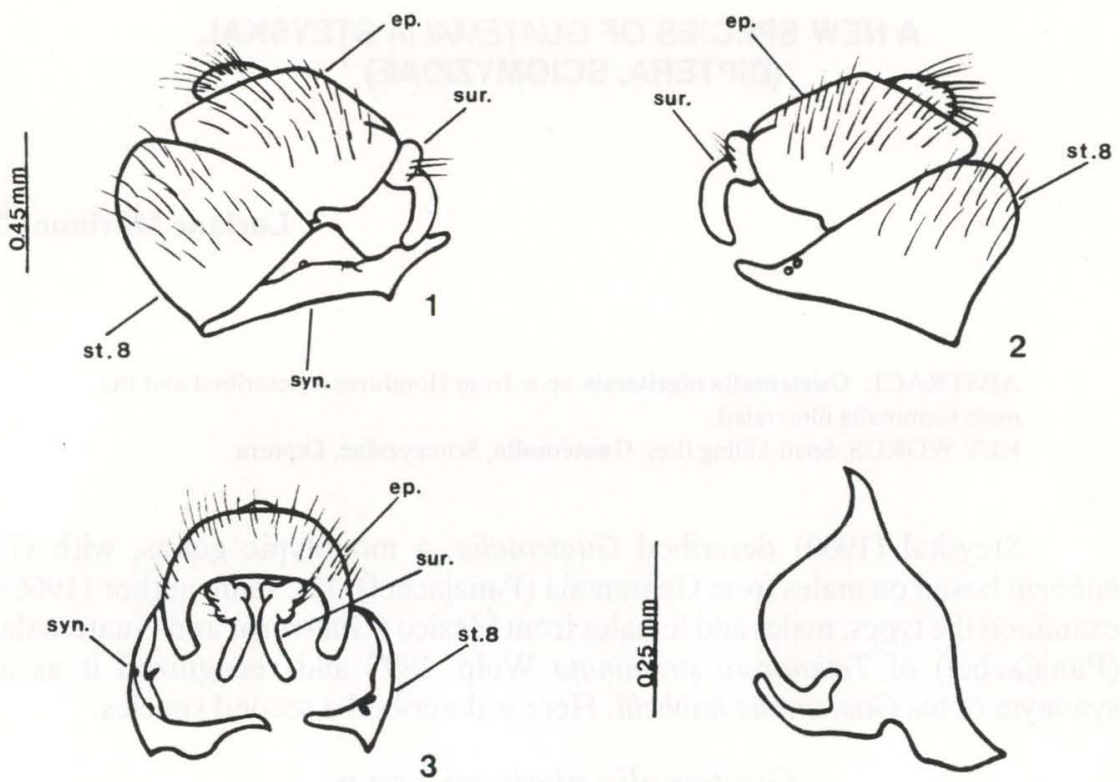

4

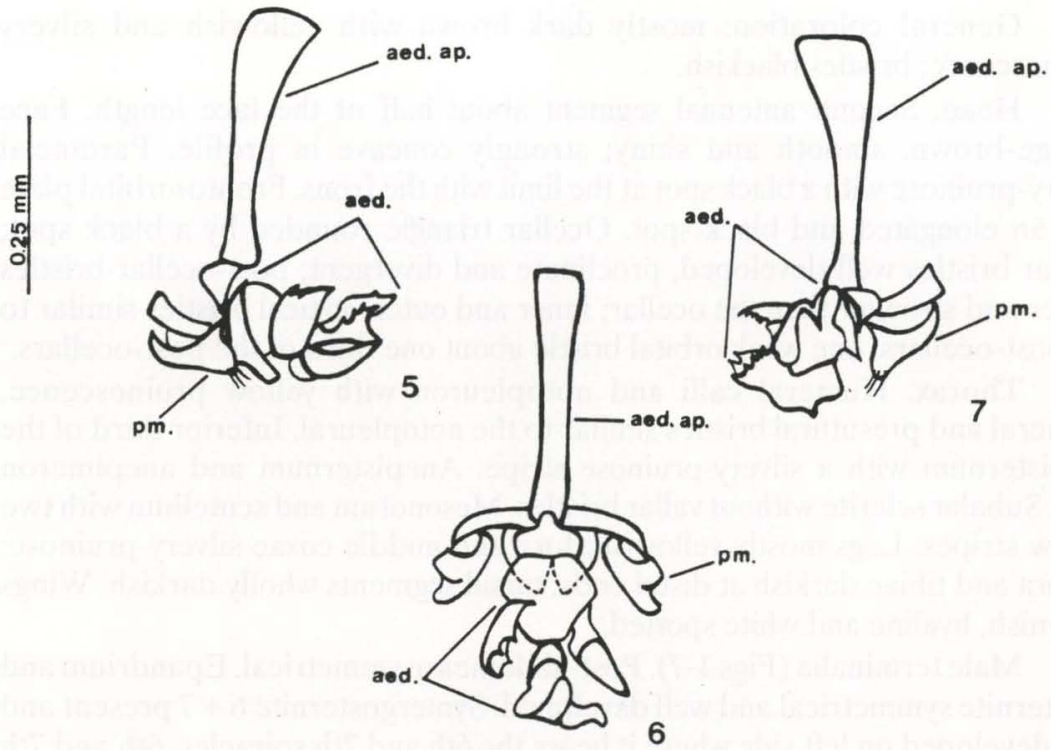

Figs 1-7. Guatemalia nigritarsis. (1-3)Left lateral, right lateral and ventral views of surstili (sur); epandrium (ep); syntergosternite 6+7 (syn); 8th sternite (st 8). (4) Lateral view of hypandrium. (5-7) Left lateral, dorsal and right lateral view of paramere (pm); aedeagus (aed); aedeagal apodeme (aed ap). 
right spiracles, very close to each other, on 8th sternite. Posterior surstyli symmetrical and well-developed. Internal sclerotized plate present. Hypandrium bare, nearly symmetric. Paramere finger-like with fine hairs. Aedeagus well-sclerotized and complex as in figs 5-7. Aedeagal apodeme well-sclerotized and elongated.

Male holotype with the following labels: "HoNDURAS: Dept./Francisco Morazan/Zambrano, nr. Tegucigalpa/Los Lago/19, 20-XI-1987, L.Knutson". "HOLOTYPE (red label)". "Guatemalia nigritarsis/Luciane Marinoni det.", National Museum of Natural History (USNM).

Comments: Guatemalia nigritarsis differs from G. straminata in the general coloration and in the male terminalia being identified by the following key:

- Face silvery pruinose; ocellar triangle rounded by a brown spot; humeral calli and notopleuron without yellow pruinescence; mesonotum and scutellum brownish; tarsal segments yellowish to brownish; posterior surstyli not globular at the base and leaf-like apically; hypandrium sinuated at the posterior margin; aedeagal apodema, in lateral view, with the distal end as wide as the proximal end (Guatemala, Mexico) .......... straminata

- Face smooth and shiny; ocellar triangle rounded by a black spot; humeral calli and notopleuron with yellow pruinescence; mesonotum and scutellum with two yellow stripes; tarsal segments wholly darkish; posterior surstyli globular at the base and slender apically; hypandrium strongly rounded at the posterior margin; aedeagal apodema, in lateral view, with the distal end larger than the proximal end (Honduras) ........... nigritarsis

\section{BIBLIOGRAPHIC REFERENCES}

STEYSKAL, G.C. 1960. New North and Central American species of Sciomyzidae (Diptera: Acalyptratae). Proc. ent. Soc. Wash. 62 (1): 33-43.

. 1966. Notes on types of some species described in Sciomyza and Tetanocera by Loew, Walker and Van der Wulp (Diptera, Sciomyzidae, Muscidae, Neriidae, Pyrgotidae). Studia ent. 8: 445-448.

Recebido em 18.V.1993; aceito em 11.VIII.1993. 